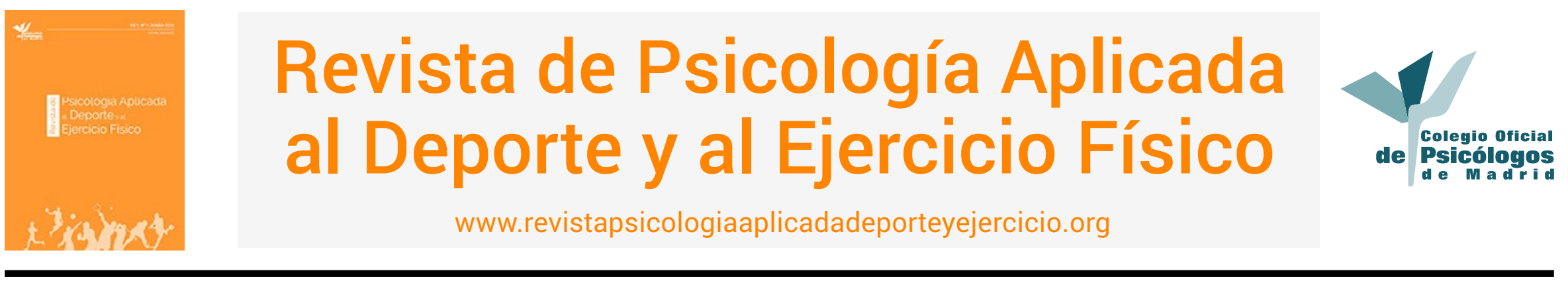

\title{
Pasado, presente y futuro del psicólogo* del deporte en el fútbol español
}

\author{
Alejo García-Naveira Vaamonde \\ MAD Lions E.C., España
}

RESUMEN: El psicólogo del deporte cuenta con una relativa larga historia en el fútbol español. Los objetivos del presente trabajo son realizar una aproximación a un mapa laboral de la actual presencia del psicólogo en los clubes de primera división y las funciones o acciones que realiza. Para ello, se ha realizado una búsqueda de información a través de medios de comunicación, páginas web y psicólogos/as del ámbito, así como una revisión de 68 trabajos sobre la temática. Los resultados indican que el psicólogo está presente en el $80 \%$ de las categorías inferiores y en un $25 \%$ de los primeros equipos de los clubes de primera división de España. Las principales funciones y acciones que realizan son la organización de escuela de padres y mediación deportiva, formación, asesoramiento e intervención con el cuerpo técnico, asesoramiento e intervención con la directiva, intervención y entrenamiento psicológico con jugadores y el equipo, entrenamiento integrado, educación, formación y entrenamiento en valores con jugadores, intervención psicológica durante la concentración y competición, desarrollo de proyecto y seguimiento académico, realización de evaluación y redacción de informes psicológicos, selección de jugadores, intervención psicológica con los servicios médicos y lesiones deportivas, retiro del jugador, estudios, formación y entrenamiento psicológico con árbitros, observación y registro de sesiones de entrenamiento y competición y divulgación e investigación en Psicología del Deporte y rol profesional del psicólogo del deporte. Se concluye que el rol del psicólogo está consolidado en el fútbol base y avanza lentamente en el fútbol profesional, en el que se requiere una labor multifunciones que aborde a la amplia población deportiva y cubra sus requerimientos desde una perspectiva científica-profesional.

PALABRAS CLAVES: Fútbol base, fútbol profesional y psicólogo deporte.

\section{Past, present and future of the sports psychologist in Spanish football}

ABSTRACT: The sports psychologist has a long history in Spanish football. The objectives of this work are to make a work map of the current presence of psychologists in the first division football clubs and their roles or actions. For this, information was collected from the media, web pages and psychologists of the field, and a review was made of 68 studies on the subject. According to the results, psychologists are present in $80 \%$ of teams in the lower categories and in $25 \%$ of the first teams of the first division clubs in Spain. The main roles and actions carried out include organization of the school for parents and sports mediation, training, advice, and intervention with the technical body, advice and intervention with the board, intervention and psychological training with players and the team, integrated training, education, training and training in values with players, psychological intervention during concentrations and competition, project development and academic monitoring, evaluation and writing of psychological reports, player selection, psychological intervention with medical services and sports injuries, withdrawal of the player, studies, training and psychological training with referees, observation and recording of training and competition sessions, and dissemination and research in Sports Psychology and the professional role of the sports psychologist. It is concluded that the role of the psychologist is consolidated in grassroots football and slowly progressing in professional football, where multifunctional work addressing the broad sports population and covering its requirements from a professional scientific perspective is required.

KEYWORDS: Base football, professional soccer, sports psychologist.

(*) La revista de Psicología Aplicada al Deporte y al Ejercicio Físico está comprometida con el uso de un lenguaje igualitario y no sexista. no obstante, en aras dela brevedad del texto y la facilidad de la lectura, en el resto del artículo se entenderá que psicólogo hace referencia a psicólogos y psicólogas, etc.

Alejo García-Naveira es Psicólogo coordinador Área I+D+i MAD Lions E.C.

La correspondencia sobre este artículo debe enviarse al autor a Avenida Julio Fuentes 11, bloque 4, 10 "A" (28660). Boadilla del Monte (Madrid).

E-mail:

(c)) EY-Nc-No Este es un artículo Open Access bajo la licencia 


\section{Passado, presente e futuro do psicólogo desportivo no futebol espanhol}

RESUMO: O psicólogo desportivo tem uma longa história no futebol espanhol. Os objetivos deste trabalho são fazer um mapa laboral da presença atual do psicólogo nos clubes da primeira divisão e as funções ou ações realizadas. Para isso, foi realizada uma pesquisa de informações através dos meios de comunicação social, páginas da web e psicólogos da área, além de uma revisão de 68 trabalhos sobre o assunto. Os resultados indicam que o psicólogo está presente em $80 \%$ das categorias mais baixas e em $25 \%$ das primeiras equipas dos clubes da primeira divisão da Espanha. As principais funções e ações realizadas são a organização da escola de pais e mediação desportiva, formação, assessoria e intervenção junto do corpo técnico, assessoria e intervenção junto da direção, intervenção e capacitação psicológica com jogadores e equipa, treino integrado, educação, formação e treino em valores com os jogadores, intervenção psicológica durante a concentração e competição, desenvolvimento de projeto e acompanhamento académico, avaliação e elaboração de relatórios psicológicos, seleção de jogadores, intervenção psicológica com serviços médicos e lesões desportivas, retirada do jogador, estudos, formação e treino psicológico com árbitros, observação e registo de treinos e sessões de competição e divulgação e pesquisa em Psicologia do Desporto e o papel profissional do psicólogo desportivo. Conclui-se que o papel do psicólogo está consolidado no futebol de base e progride lentamente no futebol profissional, o que exige um trabalho multifuncional que abranja a ampla população desportiva e cubra as suas exigências do ponto de vista científico profissional.

PALAVRAS-CHAVE: Futebol de base, futebol profissional e psicólogo desportivo.

Artículo recibido: 26/03/2018 | Artículo aceptado: 28/05/2018

El fútbol se puede considerar como el "deporte rey", siendo todo un fenómeno cultural, social, deportivo, mediático, económico e inclusive político a nivel mundial (Rodríguez, 2017). Prueba de ello es que en España existen algunos datos significativos que lo confirman. Según el Ministerio de Educación, Ciencia y Deporte (MECD, 2017), el fútbol es el deporte con mayor número de licencias federativas en España, con cerca de 942.000 (de las cuales 600.000 representan al fútbol base, 2.500 al fútbol profesional, 124.000 al fútbol de aficionados y 44.000 al fútbol femenino), seguido del Baloncesto con 355.000 aproximadamente. Otro dato importante es el poder económico del fútbol español, en el que la temporada 2016/2017, los equipos de primera división tenían un presupuesto de 2.900 millones de euros (Menchén, 2017). Por último, destacar que el fútbol es un posible ámbito laboral en España, en el cual se estima en unas 140.000 personas las que han trabajado en jornada completa durante el año 2013 (García, 2015).

Partiendo del alto impacto que posee el fútbol, y no ajeno a esta realidad, el psicólogo del deporte tiene una larga historia laboral en este contexto. Por ello, los objetivos del presente trabajo son analizar el pasado, presente y futuro del psicólogo en el fútbol español, haciendo hincapié en su presencia laboral y funciones que realiza, teniendo en cuenta los datos que existen en referencias escritas, aunque es muy probable, por el código deontológico de los profesionales de la Psicología y la confidencialidad asociada a su trabajo, que haya más psicólogos/as trabajando de los que no hay constancia manifiesta.

\section{Pasado del psicólogo del deporte en el fútbol español}

Aunque al parecer el fútbol es el deporte más estudiado entre los investigadores de habla hispana (Galindo y Arbinaga, 2017), como indica Roffé (2016), no es nada sencillo el tener información de dónde han trabajado los psicólogos/as del deporte en el fútbol, debido al anonimato, la confidencialidad profesional o falta de visibilidad, por lo que las fuentes tienen que ser varias para conseguir esta cuestión (revistas científicas, internet, periódicos, consultar a otros colegas y organizaciones de Psicología del Deporte, etc.).

Posiblemente, las primeras experiencias profesionales en la década de los años 80 hayan surgido en despachos privados y servicios no integrados en el organigrama de los clubes o federaciones, teniendo una corta duración y condiciones laborales no siempre adecuadas, lo cual demuestra la falta de una implantación real del trabajo del psicólogo del deporte aplicado en sus inicios (Cruz y Cantón, 1992).

Es con el entrenador Benito Floro en los clubes Albacete (temporadas 1989/1990 y 1991/1992) y Real Madrid (temporadas 1992/1993 y 1993/1994) donde se hace público y visible el desempeño del psicólogo dentro del primer equipo de un club de primera división (Mora, 1993). En ambos clubes, estuvieron los psicólogos Emilio Cidad y Emilio Lamparero. Como señala éste último, sin la motivación de Benito Floro por el entrenamiento psicológico hubiera sido muy difícil trabajar en el mundo del deporte profesional (Mancebo, 1992).

La primera gran experiencia profesional del psicólogo en el fútbol, al menos por el resultado deportivo, fue con la se- 
lección olímpica en Barcelona 1992, donde consiguió la medalla de oro (García y Llames, 1992). El seleccionador fue Vicente Miera y los psicólogos fueron Jesús García Barrero y Rosa Llames Lavandera. Estos profesionales, destacan el compromiso y colaboración del cuerpo técnico, fundamentalmente del entrenador, la disponibilidad de los jugadores y el tiempo y espacios adecuados utilizados.

Años más tarde surgen proyectos, unos de corta duración y escasa presencia, pero otros que sí que se han consolidado y ampliado en el tiempo, y en el que se integra el trabajo psicológico dentro de la cultura deportiva de algunos clubes de fútbol de primera división en España. Especial atención requieren el Atlético de Madrid, Real Madrid, Athletic Club de Bilbao, Deportivo de la Coruña, Villarreal, Sevilla, Getafe y Valencia.

El Atlético de Madrid, ha tenido un Departamento de Psicología del Deporte durante 14 temporadas (desde el año 2000 hasta el 2014), con los psicólogos Alejo García-Naveira y Pilar Jerez Villanueva, con participaciones tanto en el primer equipo como en el fútbol base (García-Naveira y Jerez, 2012). Anteriormente a esta etapa, hubo una experiencia con una psicóloga durante una temporada (finales de los años 90), pero que sólo se reunía con los entrenadores de la cantera, los cuales transmitían sus dudas o inquietudes en relación a sus jugadores y equipo (dato transmitido a través de uno de los coordinadores del club de esa etapa), o la presencia del psicólogo José Lorenzo González con el primer equipo durante unos meses en el año 1993, el cual lo tuvo que dejar por las presiones del contexto y la incompatibilidad personal (Miguelez, 1993).

El Real Madrid también ha tenido una larga tradición con psicólogos/as del deporte, en diferentes períodos y protagonistas, si bien no ha cuajado en el club de forma estable y ampliada a todos los equipos. Después de que el entrenador Benito Floro introdujera el rol del psicólogo del deporte, en una segunda etapa, durante 6 temporadas (desde el año 2001 hasta el 2007), estuvo el psicólogo Jose María Buceta (Interebook, 2017), tanto con la cantera como el primer equipo. Posteriormente, estuvo José Manuel Beirán, que ya había estado con Jose María Buceta, en el que también hubo muchos psicólogos que participaron en la Fundación Real Madrid trabajando con entrenadores y padres. En una tercera etapa, durante 7 temporadas (desde el año 2010 hasta el 2017), trabajó el psicólogo Javier Soriano en las categorías inferiores (Soriano, n.d.a). Y recientemente, se ha integrado la psicóloga Eva Molleja (Molleja, n.d.b).

El Athletic Club de Bilbao, ha tenido un Departamento de Psicología del Deporte durante 21 temporadas (desde el año 1996 hasta el 2017), centrado en las categorías inferiores, con la psicóloga María Ruiz de Oña (Suso, 2017).

El Deportivo de la Coruña, ha contado con la labor del psicólogo Macario Bravo durante 10 temporadas (desde el año
2006 hasta el 2016), principalmente centrado en la cantera aunque con participaciones con el primer equipo (El Desmarque, 2016). Actualmente está, en el fútbol base, la psicóloga Marisol Arias, que entró después de Macario Bravo. Años antes de eso, trabajó con el primer equipo la psicóloga Milagros Ezquerro.

Destacar la trayectoria y el excelente trabajo que se está realizando en la cantera del Villarreal desde hace 9 temporadas (desde el año 2008 hasta la actualidad), con un equipo de 9 psicólogos (Bernardo Vert, Laura Sánchez, María Cosín, Javier Garrido, Cristina Segovia, Joaquín Martínez, Álvaro Moreno y Rosa Picó), liderado por el psicólogo Eduardo Morelló (Morelló, n.d.c).

Las categorías inferiores del Sevilla desde hace 19 temporadas (desde el año 1998 hasta la actualidad), iniciado con el acuerdo establecido con el psicólogo Eugenio Pérez Córdoba de la Universidad de Sevilla, que contó con el psicólogo Miguel Morilla (CGPE, 2006; Morilla et al., 2003), actualmente tiene un equipo de 4 psicólogos/as (Juan Manuel Gamito, Mercedes Valiente, Alejandro Domínguez e Ignacio Navarro).

La escuela del Getafe desde hace 16 temporadas (desde el año 2001 hasta la actualidad) con el psicólogo José Antonio Luengo (Getafe F. C., 2016; Luengo, n.d.d).

En las categorías inferiores y primer equipo del Valencia desde hace 2 temporadas (desde el año 2016 hasta la actualidad), con diferentes etapas dentro del club, está el psicólogo José Carrascosa (Carrascosa, n.d.f). Anteriormente estuvo en el primer equipo el psicólogo Julio Figueroa, y en el año 2006/2007 el psicólogo David Peris en la cantera.

Por último, destacar varias aportaciones de psicólogos/ as en el fútbol, como las que hubo en el Betis en distintas etapas (p. ej., Eugenio Pérez Córdoba y Macarena Lorenzo Fernández); el Real Valladolid, que ha estado en primera división, que tiene al psicólogo David Rincón (lleva 13 temporadas); el Murcia, que también ha estado en primera división, que tiene desde hace varias temporadas a un grupo de psicólogos liderado por Aurelio Olmedilla Zafra; y el Elche que tuvo al psicólogo Juan González integrado dentro de los servicios médicos del club.

\section{Estado actual del psicólogo del deporte en el fútbol español}

El presente análisis se centrará, por un lado, en establecer un mapa laboral del psicólogo del deporte en los clubes de fútbol de primera división en España, tanto en los primeros equipos como en las categorías inferiores, y por otro, revisar que funciones y acciones realizan dentro de su desempeño laboral. 
Mapa laboral del psicólogo del deporte en los clubes de primera división. Continuando con las dificultades de la visibilidad del psicólogo del deporte en el fútbol español, tras realizar una revisión de las webs oficiales de los clubes de fútbol de primera división, en ninguna aparece como apartado o sub-apartado un área de Psicología del Deporte o el rol profesional del psicólogo. Por ello, la fuente principal de búsqueda ha sido el de la revisión del apartado anterior, la colaboración de otros colegas y la información que tiene el presente autor al respecto. A continuación, se presenta en la Tabla 1 un resumen de la presencia del psicólogo del deporte en el primer equipo y categorías inferiores en clubes de fútbol de primera división en España durante la temporada 2017/ 2018. Se ha valorado la mera presencia, sin entrar en las condiciones de trabajo, horarios y nivel profesional desempeñado, o la consolidación formal en el equipo.

Estos datos indican que el porcentaje de psicólogos/as que han trabajado en la temporada 2017/ 2018 en primera

Tabla 1.- Resumen de clubes de fútbol de primera división en la temporada 2017/ 2018 que cuentan o no con presencia del psicólogo del deporte en el primer equipo $\mathrm{y} / \mathrm{o}$ categorías inferiores

\begin{tabular}{lcc}
\hline $\begin{array}{l}\text { Clubes primera división de España } \\
\text { Temporada 2017/ } \mathbf{2 0 1 8}\end{array}$ & $\begin{array}{c}\text { Primer } \\
\text { equipo }\end{array}$ & $\begin{array}{c}\text { Categorías } \\
\text { inferiores }\end{array}$ \\
\hline F. C. Barcelona & No & Si \\
Real Madrid C. F. & No & Si \\
Club Atlético de Madrid & No & No \\
Athletic Club de Bilbao & No & No \\
Villarreal C.F. & No & Si \\
Real Sociedad & Si & Si \\
Sevilla F.C. & No & Si \\
Real Betis Balompié & No & Si \\
Levante U.D. & Si & Si \\
S.D Éibar & No & Si \\
Deportivo Alavés & No & Si \\
Deportivo de la Coruña & $\mathrm{Si}$ & $\mathrm{Si}$ \\
U.D. Las Palmas & No & Si \\
Getafe C.F. & No & Si \\
R. C. Celta de Vigo & $\mathrm{Si}$ & $\mathrm{Si}$ \\
Málaga C.F. & $\mathrm{Si}$ & $\mathrm{Si}$ \\
Girona F.C. & $\mathrm{No}$ & $\mathrm{No}$ \\
Valencia C.F. & $\mathrm{No}$ & $\mathrm{Si}$ \\
C. D. Leganés & $\mathrm{No}$ & $\mathrm{No}$ \\
R.C.D. Español & $\mathrm{No}$ & $\mathrm{Si}$ \\
\hline
\end{tabular}

división es de un $25 \%$ ( 5 sobre 20 clubes), mientras que en el fútbol base es del 80\% (16 sobre 20 clubes).

En cuanto a las experiencias en primera división consolidadas desde el inicio de temporada, especial atención requiere, por su trayectoria profesional, el psicólogo del deporte Joaquín Valdés, que estuvo ligado primero al Sporting de Gijón (año 2000 al 2005) y ya con Luis Enrique en el Barça B (año 2008 al 2011), en la Roma (año 2011 al 2012), el Celta de Vigo (año 2013 al 2014) y primer equipo del Barcelona (año 2015 al 2017) (Sans, 2014). Actualmente integra el cuerpo técnico del primer equipo en el Celta de Vigo, con el entrenador Juan Carlos Unzúe (Castro, 2017). Posiblemente esta sea la experiencia profesional más consolidada en primera división del fútbol español.

Por otro lado, indicar que la Real Sociedad no ha contado con un psicólogo dentro de la plantilla del primer equipo, aunque el entrenador Eusebio Sacristán utilizó a nivel personal los servicios del psicólogo experto en coaching Juan Carlos Campillo (Marín, 2016) durante el tiempo que estuvo a cargo del mismo, destituido en el mes de marzo de 2018 (El País, 2018).

Respecto a la intervención psicológica ante situaciones de "emergencia", dado los malos resultados deportivos del Málaga (estaba en puestos de descenso a segunda división), el entrenador del primer equipo José Miguel González Martín del Campo (Michel), destituido en enero de 2018, había integrado la labor del psicólogo Fernando González durante los meses de noviembre y diciembre de 2017 (Alonso, 2017). Posteriormente, como proyecto a desarrollar, el psicólogo Santiago Rivera comenzó a trabajar en la academia del club (Málaga C.F., 2018). En cuanto al Levante, también por su difícil situación deportiva en la clasificación (cuarto puesto por la "cola") contrataron a Juan Bernat, psicólogo experto en coaching que colaboraba desde enero de 2018 (Levante, 2018). También el Deportivo de la Coruña, cuando estaba en puestos de descenso, integraron al psicólogo Ricardo de la Vega (Centeno, 2018).

Y por último, fuera del análisis de los clubes de fútbol de primera división en España, es significativo que Julen Lopetegui, exseleccionador Nacional de Fútbol de España, trabajaba a nivel personal con el psicólogo experto en coaching Juan Carlos Campillo (Moñino, 2016).

Funciones y acciones del psicólogo del deporte en el fútbol. A modo de contextualizar la labor del psicólogo del deporte en general, destacar los trabajos de Cantón (2010, 2016), en el que se resume los ámbitos de actuación (deporte de rendimiento, deporte de base e iniciación, deporte de ocio y tiempo libre, deporte en poblaciones con necesidades específicas y organizaciones deportivas), funciones (evaluación y diagnóstico, planificación y asesoramiento, interven- 


\section{Tabla 2. Principales funciones y acciones que realizan los psicólogos/as del deporte en el fútbol}

Escuela de padres y madres y mediación deportiva

Formación, asesoramiento e intervención psicológica con el cuerpo técnico
Álvarez y Chico (2016); García-Naveira y Jerez (2012); Hernández (2016); Morilla et al. (2003); Ortiz (2017); Villalonga, Garcia-Mas, De las Heras, Buceta y Smith (2015).

Álvarez y Chico (2016); Castillo, Ramis, Cruz y Balaguer (2015); D’Amico, Cañizares y Sánchez (2017); García y Llames (1992); García-Naveira (2017); García-Naveira y Jerez (2012); Gómez-Espejo, Aroca, Robles-Palazón y Olmedilla (2017); Hernández (2016); Martí (2002); Martín (2003); Morilla et al. (2003); Olmedilla, García y Garcés de los Fayos (1998); Olmedilla, Ortín, Andréu y Lozano (2004); Ortiz (2017); Rivas y Aragón (2003); Villalonga et al. (2015).

Álvarez y Chico (2016); García-Naveira y Jerez (2012); Vives y Garcés de los Fayos (2003).

Asesoramiento e intervención con la directiva Álvarez y Chico (2016); Andreu (2003); Carmona, Guzmán y Olmedilla (2015); De la Vega, R. (2003); D'Amico et al. (2017); García y Llames (1992); García-Naveira y Jerez (2012); Godoy-Izquierdo, Vélez y Pradas (2009); Gómez-Espejo, Aroca et al. (2017); Hernández (2016); Martín (2003). Mora, Zarco y Blanca (2001); Morilla et al. (2003); Navarrón, Godoy-Izquierdo, Vélez, Ramírez-Molina y Jiménez-Torres (2017); Olmedilla y Dominguez-Igual (2016); Olmedilla, Ortega, Andreu y Ortín (2010); Olmedilla, Ortega, Ortín y Andreu (2008); Rivas y Aragón (2003); Roffé (2016).

Entrenamiento psicológico con el equipo

Álvarez y Chico (2016); Chicau, Silva y Palmi (2012); D’Amico et al. (2017); García-Naveira y Jerez (2012); Hernández (2016); Martín (2003); Morilla et al. (2003); Rivas y Aragón (2003); Roffé (2016).

Entrenamiento psicológico integrado

De la Vega (2003); García-Naveira y Jerez (2012); Morilla et al. (2003); Morilla, Utrilla, Antón y Rivera. (2009).

Educación, formación y entrenamiento psicológico en valores con jugadores

Álvarez y Chico (2016); Cruz, Boixadós, Torregrosa y Mimbrero (1996); Escolano-Pérez, Herrero-Nivela y Echeverría-Expósito (2014); García-Naveira y Jerez (2012); Gimeno, Sáenz, Vicente y Aznar (2007); Gómez-Espejo, Aroca, et al. (2017); González, García, Pastor y Contreras (2011); Lamoneda y Huertas (2017); Martí (2002); Morilla et al. (2003); Ortiz (2017); Rivas y Aragón (2003).

Intervención psicológica durante la competición

García y Llames (1992); Ramírez y Rodríguez (2015); Roffé (2016).

Intervención psicológica durante la concentración de partido o competición

García y Llames (1992); Hernández (2016); Ramírez y Rodríguez (2015); Roffé (2016).

Proyecto y seguimiento académico

García-Naveira y Jerez (2012); Hernández (2016); Martín (2003); Morilla et al. (2003); Morilla et al (2009): Villalonga et al. (2015)

Evaluación y redacción de informes psicológicos

García y Llames (1992); García-Naveira y Jerez (2012).

Selección de jugadores Llames (1999); Martí (2002).

Intervención psicológica con los servicios médicos y lesiones deportivas

García-Naveira y Jerez (2012); Gómez-Espejo, Álvarez, Abenza y Olmedilla (2017); Hernández (2016); Martín (2003); Olmedilla, García y Martínez (2006); Olmedilla, Ortega y Abenza (2007); Prieto, Ortega, Garcés de los Fayos y Olmedilla (2014).

Retiro del jugador

Duque-Ingunza y Dosil (2017); Lorenzo y Bohórquez, (2013); Lorenzo y Bueno (2012).

Estudios, formación y entrenamiento psicológico con árbitros

Alonso-Arbiol, Arratibel y Gómez (2008); Botella y Palacios (2002); Brandão, Serpa, Krebs, Araújo y Machado (2011); De la Vega, Carrasquilla, Ortiz, Ruiz-Barquín y Armenteros (2017); Gómez y Botella (2005); González, (2006); Marrero y Gutiérrez (2002); Rodríguez, Salazar y Arroyo (2009).

Observación y registro del rendimiento en sesiones de entrenamiento y competición

García-Naveira y Jerez (2012); Gómez-Espejo et al. (2017); Morilla et al. (2003); Vives y Garcés de los Fayos (2003); Villalonga et al. (2015).

Gómez-Espejo, Aroca et al. (2017); Martí (2002).

Divulgación e investigación en Psicología del Deporte y rol profesional del psicólogo del deporte 
ción, educación y/o formación e investigación) y la población deportiva con la que se trabaja (directores deportivos, cuerpo técnico, deportistas, árbitros, padres, etc.).

También, como señala García-Naveira (2010, 2016), los objetivos de desempeño del psicólogo del deporte son el logro de resultados, mejora del rendimiento y la salud, desarrollo profesional y del talento, crecimiento personal, creación de equipos de alto rendimiento, entre otros, mientras que para su consecución, dentro de un proceso de evaluación-intervención, se emplean diferentes modelos, teorías y estrategias psicológicas como son las de orientación cognitiva-conductual, psicodinámica, coaching, mindfulness, PNL, etc.

Partiendo de estas cuestiones, para realizar un análisis de las funciones y acciones que realizan los psicólogos/ as del deporte en el fútbol, se han revisado 68 trabajos de investigación aplicada, experiencias profesionales y temas relevantes a nivel práctico de revistas científicas españolas en Psicología del Deporte (Revista de Psicología del Deporte, Revista Iberoamericana de Psicología del Ejercicio y el Deporte, Cuadernos de Psicología del Deporte y Revista de Psicología Aplicada al Deporte y al Ejercicio Físico) y otras revistas genéricas de Psicología (Informació Psicológica, Papeles del Psicólogo y Acción Psicológica, etc.). En la Tabla 2 , se presenta un resumen de las principales líneas de trabajo del psicólogo del deporte en el fútbol.

Además, los psicólogos/as del deporte y otros profesionales de las Ciencias del Deporte, han contribuido a la comprensión comportamental del juego y de situaciones deportivas que se dan en el fútbol. Prueba de ello son los estudios como el efecto de marcar primero y la ventaja de jugar en casa (Sampedro y Prieto, 2012), la eficacia de los saques de esquina (Ardá, Maneiro, Rial, Losada López y Casal, 2014), la consecución de un gol en el último tramo del primer periodo (Roffé, De la Vega, Garcia-Mas y Llinás, 2007), la evolución y transiciones del ataque en el fútbol de élite (Barreira, Garganta, Castellano, Prudente y Anguera, 2014; Casal, Losada y Ardá, 2015), el análisis de los perfiles de rendimiento en función de las acciones del juego de los equipos ganadores (Vales, Blanco, Areces y Arce, 2015) y la relación entre indicadores de rendimiento y el éxito en el fútbol profesional (Castellano, 2018).

Por último, destacar la aportación de diferentes autores en temas que son relevantes en el ámbito de la Psicología del Deporte y el fútbol, como son la transición de junior al fútbol profesional (Chamorro, Torregrosa, Sánchez y Amado, 2016; Pazo, Sáenz-López y Fradua, 2012), el análisis de la carga física y psicológica en jugadores profesionales (Calahorro, Torres y Lara, 2014; Gómez-Díaz, Pallarés, Díaz y Bradley, 2013), el conocimiento táctico y toma de decisiones en jóvenes jugadores de fútbol (Escolano-Pérez, Herrero-Nivela y Echeverría-Expósito, 2014: Rivas y Aragón, 2003), la evaluación e intervención en los porteros de fútbol (Bohórquez y Checa, 2017; Estrada, Silva, Pérez-Córdoba, Borrego y Cantón, 2017), entre otras.

\section{Discusión}

Como se ha podido observar en el presente trabajo, el "mundo del fútbol" puede ser un nicho de mercado para los psicólogos/as del deporte debido al elevado número de licencias y el poder económico con el que cuenta, principalmente en los clubes de primera división, que avanza muy lentamente su presencia en el fútbol profesional (25\% de los equipos de primera división) y que está bastante instaurado en el fútbol base $(80 \%$ de los clubes de primera división). A pesar de ello, en ocasiones, el Departamento de Psicología está estructurado en las categorías inferiores, aunque pueden ofrecer sus servicios con el primer equipo cuando se le requiere, principalmente desde una perspectiva del déficit y no tanto del entrenamiento psicológico para la mejora del rendimiento y la salud de los deportistas (García-Naveira y Jeréz, 2012; Morilla et al., 2003). Además, muy probablemente hay muchos jugadores y entrenadores que cuentan con psicólogos/as externos al club, de manera individual y de los que no hay constancia escrita por el cumplimiento del código deontológico y el respeto a la confidencialidad.

En relación a estos datos, como señala el MECD (2017), destacar que el mayor número de licencias se encuentra en el fútbol base (64\%) y en menor medida en el fútbol profesional $(0.26 \%)$. Esto puede indicar que la mayor probabilidad de puestos de trabajo para el psicólogo del deporte se encontraría en las categorías inferiores, aunque económicamente a veces no sea lo mejor remunerado, ya que el presupuesto anual de los clubes se centra principalmente en el fútbol profesional (Menchén, 2017).

Además, indicar que el psicólogo realiza una labor multifunciones dentro de un club de fútbol, abordando a la población deportiva y sus requerimientos desde una perspectiva científica-profesional, cuestiones que se irán comentando en la discusión. Es por ello que este puede ser un buen momento para reflexionar y analizar la situación actual del psicólogo del deporte en el fútbol español con los objetivos de terminar de asentarse y progresar en la profesión. A continuación se aborda estas cuestiones destacando el fútbol profesional, fútbol base, trabajo psicológico con el jugador y equipo y otras cuestiones relacionadas con la profesión del psicólogo. 


\section{Fútbol profesional}

En el fútbol profesional, posiblemente el entrenador es la clave para que el psicólogo acceda al primer equipo, tal y como se ha citado en diferentes experiencias (Alonso, 2017; Castro, 2017; García y Llames, 1992; Mancebo, 1992; Marín, 2016; Moñino, 2016). El psicólogo del deporte dentro del cuerpo técnico, es el posicionamiento más eficaz para su desempeño profesional (Olmedilla et al., 1998). Debido a que el fútbol profesional está condicionado por los resultados deportivos, los objetivos de intervención del psicólogo del deporte tienen que centrarse en el logro de resultados, mejora del rendimiento y salud a través del entrenamiento psicológico de las personas (García-Naveira, 2010, 2016).

A partir de este punto pueden surgir las siguientes preguntas: ¿Qué busca el entrenador? ¿Qué necesita? ¿Cómo se puede llegar a integrar el cuerpo técnico? Si los psicólogos/ as son capaces de responder a estas preguntas, posiblemente estarán más cerca de la inserción laboral en el fútbol profesional y adaptar su labor a los requerimientos deportivos. Futuros trabajos podrían iluminar estas cuestiones.

Otro escenario diferente es cuando un equipo obtiene malos resultados durante la temporada y las urgencias deportivas hacen que el club y/o entrenador se aferre al psicólogo como una posible solución a "todos los males" que tiene el equipo, como ha sido el caso del Málaga (Alonso, 2017), el Levante (Levante, 2018) y el Deportivo de la Coruña (Centeno, 2018), con un final dispar entre ellos, ya que solo el Levante salvó la categoría. Al respecto, indicar que el entrenamiento psicológico tiene que partir desde la planificación y organización (p. ej., pre-temporada), y no de situaciones límites, donde la probabilidad de éxito suele ser menor, tal y como se ha quedado establecido en estos casos. Ahora bien, sin "vender humo" sobre los resultados y sabiendo que se requiere un trabajo psicológico riguroso y en condiciones adecuadas, el psicólogo deberá de valorar, a partir de la demanda, su formación y experiencia profesional, si se expone o no ante esa situación. En este sentido, la acreditación profesional de Experto en Psicología del Deporte del Consejo General de la Psicología de España (CGPE, 2018), garantiza unos mínimos necesarios para poder actuar con eficiencia en el deporte, siendo esta un referente para los psicólogos/ as y las propias instituciones al contratar un profesional de esta área.

\section{Fútbol base}

Probablemente en las categorías inferiores, la presencia del psicólogo del deporte en los clubes dependa principal- mente del director deportivo, la tradición, filosofía y metodología de trabajo de la entidad (García-Naveira y Jeréz, 2012; Morilla et al., 2003). Es por ello que la Psicología de las Organizaciones cobra especial interés para el desarrollo de la entidad deportiva e inserción laboral. Por ejemplo, trabajar con la directiva para que estos obtengan un mayor rendimiento y bienestar (desarrollo del liderazgo, toma de decisiones, resolución de conflictos, reducir el estrés, etc.), beneficia a la persona, la marcha del club y al psicólogo, ya que en este último caso "ven de primera mano" su trabajo (Álvarez y Chico, 2016; García-Naveira y Jerez, 2012; Vives y Garcés de los Fayos, 2003). Otras cuestiones a profundizar en futuros trabajos son el establecimiento de la misión, valores y objetivos del club, selección y desarrollo del talento de los empleados, mejora de la imagen y comunicación del club (interna y externa), etc.

Otro tema relevante dentro de la organización, la cultura y metodología deportiva de trabajo para el desarrollo de un proyecto deportivo son la filosofía y los principios en los que estos se basan, definiendo qué perfil de jugador se quiere y cuál es el camino a seguir para conseguirlo. Por ejemplo, fundamentos como, el jugador es el proyecto, la potencialidad del jugador, el jugador-persona, el jugador-pensante, el entrenamiento y formación integral y el entrenamiento-formación interna (dentro del contexto deportivo) y externa (fuera del contexto deportivo) del jugador (ver García-Naveira-Jerez, 2012 y Vives y Garcés de los Fayos, 2003).

Además de la labor organizacional del psicólogo, los objetivos principales del club a conseguir son que los jugadores de fútbol base lleguen al fútbol profesional (entre el 1-3\% de la población) y obtengan un futuro prometedor en la vida tras su retirada o abandono deportivo (García-Naveira y Jerez, 2012; Morilla et al., 2003). Para ello, diferentes autores establecen que las funciones fundamentales del psicólogo son de educar, formar y entrenar en valores para el éxito personal y deportivo del jugador, observando la necesidad de formar primero personas y luego las habilidades deportivas del jugador (Álvarez y Chico, 2016; Cruz et al., 1996; Escolano-Pérez et al. 2014; García-Naveira y Jerez, 2012; Gimeno et al. 2007; Gómez-Espejo, Aroca et al., 2017; González et al., 2011; Lamoneda y Huertas, 2017; Martí, 2002; Morilla et al., 2003; Ortiz, 2017; Rivas y Aragón, 2003; Roffé y Braco, 2003).

Para ello, es importante establecer un modelo de trabajo para desarrollar el talento de los jugadores, como por ejemplo el Programa Integral Deportivo (PID; García-Naveira y Jerez, 2012) en el que el jugador se desarrolla a partir de un entrenamiento sistemático en 4 áreas: actividad deportiva, familia y relaciones sociales, actividad académica y educación-formación. 
En relación a estos puntos, destacar la importante función desempeñada por el psicólogo con la formación, asesoramiento e intervención psicológica con el cuerpo técnico como líderes deportivos y principales gestores a nivel psicológico del jugador y equipo, por ejemplo en el entrenamiento de habilidades de comunicación, motivación o autorregulación emocional (Álvarez y Chico, 2016; Cantón, Checa y Budzynska, 2013; Castillo et al., 2015); D’Amico et al., 2017; García y Llames, 1992; García-Naveira, 2017; García-Naveira y Jerez, 2012; Gómez-Espejo, Aroca et al., 2017; Hernández, 2016; Martí, 2002; Martín, 2003; Morilla et al., 2003; Olmedilla et al., 1998; Olmedilla et al., 2004; Ortiz, 2017; Rivas y Aragón, 2003; Villalonga et al., 2015), con las escuelas de padres y mediación deportiva, como por ejemplo en la definición del rol y funciones de los padres y madres, así como el desarrollo del liderazgo hacia sus hijos (Álvarez y Chico, 2016; García-Naveira y Jerez, 2012; Hernández, 2016; Morilla et al., 2003; Ortiz, 2017; Villalonga et al., 2015) y en el desarrollo de proyectos académico de los jugadores, como por ejemplo, el seguimiento de las notas y premiar las buenas calificaciones (García-Naveira y Jerez, 2012; Hernández, 2016; Martín, 2003; Morilla et al., 2003; Morilla et al. 2009: Villalonga et al., 2015)

Debido a la edad de los jugadores, principal atención requiere la adolescencia debido a los múltiples cambios que padece (Roffé y Braco, 2003) y su transición al fútbol profesional o en su defecto al deporte adulto (Chamorro et al., 2016; Pazo et al., 2012). El trabajo psicológico se tiene que adaptar a la edad biológica del jugador, sus necesidades y circunstancias (García-Naveira y Jerez, 2012; Martí, 2002; Morilla et al., 2003; Vives y Garcés de los Fayos, 2003). Por ejemplo, en categorías de edades tempranas (chupetín o querubín y prebenjamín) hay que centrarse principalmente en el desarrollo de la autonomía y juego individual, para posteriormente ir poco a poco integrando el juego colectivo y la percepción del otro, ya que a estas edades (5-8 años) su mente no suele estar preparada para el trabajo en equipo.

Desde la perspectiva del club y el trabajo del psicólogo, tiene que haber un proyecto temporalmente establecido para realizar estas y otras acciones, por ejemplo, con un margen de acción de 10 años (Martí, 2002; Vives y Garcés de los Fayos, 2003). En mi opinión, los ciclos tienen que ser de 4 y 8 años, con indicadores para valorar su evolución (rendimiento y resultados deportivos individuales/ grupales, pruebas físicas, resultados académicos, evaluación psicológica...). Además, el futuro deportivo tiene que estar en el fútbol base para generar sus propios jugadores debido a la situación económica de algunos clubes y el impacto deportivo y económico que puede tener, con la necesidad de dotar con un mayor presupuesto y recursos (inversión) esta sección del club.

\section{El trabajo psicológico con el jugador y equipo}

La intervención y entrenamiento psicológico individual con el jugador es una de las acciones del psicólogo más habituales y con mayor trayectoria en el fútbol (Álvarez y Chico, 2016; Andreu, 2003; Carmona et al., 2015; De la Vega, 2003; D’Amico et al., 2017; García y Llames, 1992; García-Naveira y Jerez, 2012; Godoy-Izquierdo et al., 2009; Gómez-Espejo, Aroca et al., 2017; Hernández, 2016; Martín, 2003; Mora et al., 2001; Morilla et al., 2003; Navarrón et al., 2017; Olmedilla y Dominguez-Igual, 2016; Olmedilla et al., 2010; Olmedilla et al., 2008; Rivas y Aragón, 2003; Roffé, 2016). Los objetivos generales se centran en optimizar y preparar psicológicamente al deportista para que mejore su rendimiento y salud. Por ejemplo, se entrena la motivación, concentración, confianza y competir bajo situaciones de estrés.

Atención especial requieren los jugadores foráneos (García-Naveira y Jerez, 2012; Morilla et al., 2003), en el cual pueden estar lejos de sus familias y solos, en el que las diferencias culturales pueden estar presentes y dificulte su adaptación. Algunas cuestiones a atender son la estancia (residencia o vivienda), colegio/ estudios (para el jugador o sus hijos) e inmersión cultural (p. ej., costumbres, idioma, convivencia y normas).

Otra cuestión relevante, menos habitual y con menor trayectoria en el fútbol es la intervención y entrenamiento psicológico grupal (Álvarez y Chico, 2016; Chicau et al., 2012; D’Amico et al., 2017; García-Naveira y Jerez, 2012; Hernández, 2016; Martín, 2003; Morilla et al., 2003; Rivas y Aragón, 2003; Roffé, 2016). Los objetivos generales se centran en optimizar y preparar psicológicamente al equipo para que mejore su rendimiento y ambiente de trabajo. Por ejemplo, se entrenan diferentes variables grupales como el liderazgo, establecimiento de metas comunes, normas compartidas, estatus y roles.

Realizando una valoración al respecto, se observa la necesidad de realizar más trabajos y profundizar sobre la temática. Desde mi experiencia profesional, percibo cierta dificultad del entrenador de posibilitar al psicólogo el acceso al grupo, tal vez debido a una necesidad de control, desconocimiento del rol del psicólogo y falta de trabajo interdisciplinar, especialmente en equipos semi-profesionales o profesionales. Aunque el trabajo con el equipo puede ser importante y necesario según las necesidades establecidas, el psicólogo no tiene que obsesionarse por esta cuestión. No es necesario conquistar siempre "al equipo", también se puede entrenar al cuerpo técnico para que realice actividades y dinámicas grupales (intervención indirecta), dentro de un proceso de evaluación pre y post intervención, siendo el psicólogo en estos casos, un buen observador externo de la acción. 
Esta línea de actuación suele ser bien recibida por el cuerpo técnico.

En cuanto al entrenamiento integrado, se deben plantear actividades que integren las exigencias técnicas, tácticas, físicas y psicológicas similares a las que se enfrentará el futbolista durante un partido o en función de los objetivos establecidos para dicha sesión (De la Vega, 2003; García-Naveira y Jerez, 2012; Morilla et al., 2003; Morilla et al., 2009). En concreto, se busca entrenar mentalmente al jugador a través de un trabajo integrado sistemático y riguroso de campo. Al respecto, pueden surgir dos líneas de actuación, no necesariamente opuestas. Por un lado, el entrenamiento integrado desde el propio entrenamiento (p. ej., trabajar con el entrenador para que este establezca objetivos psicológicos, planificar y organizar los entrenamientos con contenido psicológico y gestionar los entrenamientos según su evolución), como por ejemplo, generando situaciones estresantes para conseguir la adaptación del organismo (García-Naveira y Jerez, 2012). Por otro, se encuentran las actividades organizadas fuera del propio entrenamiento del entrenador con tareas específicas para mejorar alguna variable psicológica como la concentración (De la Vega, 2003; Morilla et al., 2013; Morilla et al., 2009). En mi opinión, lo mejor aceptado y más eficaz es cuando se trabaja con el entrenador para que este planifique y gestione a los deportistas, mientras que las tareas específicas pueden ser un buen complemento para cuando haya algo muy concreto a evaluar y/o entrenar.

Respecto al trabajo psicológico en concentraciones, es una parcela relevante que los psicólogos tienen cubrir, mostrar su eficacia e investigar (García y Llames, 1992; Hernández, 2016; Ramírez y Rodríguez, 2015; Roffé, 2016). Los momentos previos a la competición y/o el tener una buena estancia durante días o semanas, puede ser determinante para el rendimiento y salud del equipo, en el que además se observan pocos trabajos al respecto. La adaptación a la concentración y convivencia, el trabajo para mejorar la cohesión y la atención individual en los casos que se requieran, son algunas cuestiones que se abordan.

El trabajo psicológico durante las competiciones es otro momento deportivo en el que el psicólogo debe de estar disponible para realizar intervenciones de campo (García y Llames, 1992; Ramírez y Rodríguez, 2015; Roffé, 2016), en el que se observa la necesidad de profundizar en esta temática y realizar estudios al respecto. El acompañamiento en estas situaciones, por un lado, puede posicionar el rol profesional del psicólogo, y por otro, ser útil a los jugadores y al cuerpo técnico. Por ejemplo, desde una perspectiva algo más pasiva, y no por ello menos importante, estando presente como apoyo, transmitiendo estabilidad y confianza durante todo el proceso pre y pos competitivo (hotel, desplazamiento, ves- tuario, descanso del partido, tras finalizar el partido, etc.), así como contribuir de forma activa a la adaptación a la competición (p. ej., ansiedad pre-competitiva, aislamiento de presión ambiental, autocofianza y expectativas de éxito).

Otro tema a progresar en relación a este punto es la obtención de una licencia federativa para el psicólogo del deporte, tal y como sucede en Italia, al igual que la poseen los entrenadores, preparadores físicos y servicios médicos. Los objetivos son poder estar presentes en los banquillos como reconocimiento profesional, sin la necesidad de tener un permiso como utilero u otro rol, y poder estar disponible para realizar alguna intervención de campo (p. ej., estilo de comunicación del entrenador y control del estrés de los jugadores en el banquillo). Futuras acciones colegiales se están llevando a cabo para avanzar sobre esta cuestión.

Destacar que es necesario realizar una evaluación del programa psicológico aplicado para valorar su eficacia (García y Llames, 1992; García-Naveira, 2017; Olmedilla y Dominguez-Igual, 2016). Para ello, se requiere establecer previamente unos indicadores de rendimiento que se valorarán durante su seguimiento o finalización del mismo (rendimiento y resultados deportivos, rendimiento académico, hábitos saludables, variables psicológicas, conductas observables, valoración subjetiva con una escala tipo Likert sobre la satisfacción del programa, etc.). En relación a este punto, destacar la observación y registro del rendimiento en las sesiones de entrenamiento y competición dentro de un proceso pre y pos intervención (García-Naveira y Jerez, 2012; Gómez-Espejo, Aroca et al., 2017; Morilla et al., 2003; Vives y Garcés de los Fayos, 2003; Villalonga et al., 2015).

Otra cuestión a atender es la evaluación psicológica e informes individual y /o grupal que en ocasiones solicita la institución que contrata al psicólogo (García y Llames, 1992; García-Naveira y Jerez, 2012). En primer lugar, hay que comprender que el cliente es el jugador o entrenador y no el club, aunque sea este el que pague al profesional. Esta cuestión hay que dejarla clara desde el principio a todas las personas implicadas (p. ej., coordinador y entrenador) para que no haya malos entendidos y se defina qué información se puede transmitir y quién la debería de dar (p. ej, el propio cliente). Por ello, el psicólogo tiene que cumplir con el código deontológico y la legalidad (p. ej., confidencialidad y protección de datos del cliente). Al respecto, más trabajos se requieren para establecer protocolos de actuación ante esta y otras posibles situaciones que se pueden dar en el contexto deportivo.

En relación a los procesos detección de talento y selección de jugadores, la evaluación psicológica tiene que complementar los métodos tradicionales, en los que habitualmente se centran en la figura del ojeador y la revisión médica (Llames, 1999; Martí, 2002). La tendencia tiene que ir encaminada ha- 
cia una metodología interdisciplinar (entrenador, preparador físico, psicólogo, médico y ojeador) que ofrezca indicadores deportivos y comportamentales que permitan aumentar la probabilidad de acertar en la elección del jugador, con su correspondiente beneficio deportivo y económico. Para una revisión sobre la temática desde una perspectiva psicológica, ver los trabajos de Ruiz-Barquín (2015) y Sánchez-Gombau y Guillén (2015).

El trabajo con los servicios médicos y lesiones deportivas es importante desde un punto de vista de la salud y el rendimiento deportivo, antes (vulnerabilidad psicológica), durante (estado psicológico óptimo y adherencia a la rehabilitación) y después (prevención de lesiones y reincorporación deportiva) de la lesión (García-Naveira y Jerez, 2012; Gómez-Espejo, Álvarez et al., 2017; Hernández, 2016; Martín, 2003; Olmedilla et al., 2006; Olmedilla et al., 2007; Prieto et al., 2014). Además de atender al deportista, las sesiones médicas ( $p$. ej., semanalmente) y conversaciones regulares con otros profesionales es básica para un mayor rendimiento interno y tratamiento del lesionado desde una perspectiva interdisciplinar (García-Naveira, 2016).

En cuanto al retiro del jugador, ya que llega antes o después (edad, lesiones, decisión personal, etc.), ésta de por sí puede ser una situación traumática en la que habitualmente los deportistas no están preparados para ella. (Duque-Ingunza y Dosil, 2017; Lorenzo y Bohórquez, 2013; Lorenzo y Bueno, 2012). Sin profundizar en la temática, las carreras duales, donde el deportista se prepara deportivamente y a nivel académico, suele ser el mejor camino para crecer como persona y deportista, así como planificar una salida laborar posterior cuando se retire del deporte (Chamorro et al., 2016; Pazo et al., 2012)

Por último, destacar también el manejo y gestión del contexto del jugador -prensa, fama, dinero, relaciones, etc.- (Rivas y Aragón, 2003) y el desarrollo de la identidad y pertenencia al equipo para que el jugador quiera seguir en el club, esté satisfecho, tenga una valoración positiva, perciba progreso, prestigio, etc. (García-Naveira y Jerez, 2012; Rivas y Aragón, 2012).

\section{Otras cuestiones relacionadas con la profesión del psicólogo}

En cuanto a la inserción laboral del psicólogo, más allá del peso que pueda tener el entrenador y la directiva en la contratación y estabilidad laboral, el desarrollo profesional en un club se puede dividir en tres etapas (García-Naveira y Jerez, 2012): 1) Inserción (introducción en el club y primeras experiencias); 2) Implementación dentro de la estructura depor- tiva y funcionamiento mantenido en el tiempo (integración del trabajo psicológico dentro de la planificación general del club durante varias temporadas) y 3) Transformación cultural (la Psicología del Deporte como parte de la metodología de trabajo y valores del club).

Al respecto, destacar que lleva tiempo construir un proyecto de Psicología del Deporte (años) y es fácil desarmarlo (días). Aunque puede formar parte de un club sin un proyecto deportivo general consolidado (p. ej., cambio regular de directores deportivos o que no tengan un modelo de trabajo establecido), es importante que el rol del psicólogo del deporte esté asociado a la filosofía del club y no tanto a una persona (entrenador o director deportivo), siendo deseable que en todo caso sean ciclos de cambios de profesionales en la entidad (psicólogo por psicólogo), pero no de la Psicología del Deporte (que se mantenga siempre el área o departamento).

Es importante conocer qué piensan los entrenadores sobre la Psicología del Deporte y el psicólogo del deporte, sobre todo porque la mayoría de ellos no ha tenido experiencias profesionales previas con un psicólogo, aunque piensan que les podría venir bien para potenciar su labor (Olmedilla et al., 1998). Esto puede significar que hay desconocimiento por el trabajo del psicólogo, interés por su aportación y cierta resistencia frente a lo que es más habitual en el fútbol (p. ej., médico, fisioterapeuta y preparador físico). Por ello, se requiere realizar un muy buen marketing y promocionar el trabajo del psicólogo, ya que en principio parece que la receptividad va a ser positiva, dar visibilidad a la eficacia del mismo y trabajar de forma interdisciplinar con otros profesionales. También hay que responder a las demandas que desde el contexto deportivo se hagan, con resultados acordes a la profesionalización (acreditación de Experto en Psicología del Deporte) y no ir dando respuesta a cuestiones ajenas a las necesidades del deporte o requerimientos de los protagonistas.

En relación al trabajo interdisciplinar y reuniones regulares de trabajo con otros profesionales (director deportivo, coordinador, entrenador, preparador físico, médico, fisioterapeuta, nutricionista, psicólogo, etc.), destacar que representa la base del éxito personal y deportivo, ya que la mejora del rendimiento, formación, salud y bienestar de los deportistas es multifactorial (García-Naveira y Jerez, 2012; Morilla et al., 2003). Para ello, se requiere organizar y planificar el trabajo psicológico, informar a otros profesionales, integrarlo dentro de la planificación global deportiva y coordinar su ejecución, y por último, distribuir los tiempos y espacios de trabajo (García y Llames, 1992; García-Naveira, 2017; García-Naveira y Jerez, 2012; Martí, 2002; Rivas y Aragón, 2003; Vives y Garcés de los Fayos, 2003). 
La visibilidad del psicólogo del deporte tiene que mejorar, ya que como se ha ido comentando en la introducción, el localizar a los psicólogos/as que trabajan en el fútbol requiere de una ardua labor (Roffé, 2016). Es por ello que el psicólogo para seguir afianzándose laboral y profesionalmente requiere de una mayor visibilidad, principalmente desde los medios de difusión del club, como puede ser en el organigrama que aparece en la web, noticias, newsletter, etc. Algunos ejemplos al respecto son los del Getafe (Getafe C. F., 2016) y Málaga (Málaga C. F., 2018) en los cuales han salido noticias reflejadas sobre la actividad del psicólogo, cuestión importante siempre y cuando se cumpla el código deontológico y la confidencialidad, que podría convertirse en una entendible "limitación" de dicha visibilidad.

Al respecto, es importante estar dentro y aparecer visiblemente en el organigrama de la entidad deportiva (Hernández, 2016), aunque otra cuestión a debatir es dónde se posiciona el psicólogo. Posiblemente el referente que ofrece una mejor estructura, profesionalidad y estabilidad laboral para el psicólogo sean los servicios médicos frente a ser un departamento independiente (García-Naveira, 2016; García-Naveira y Jerez, 2012), aunque en ambas opciones existen ventajas y desventajas que el psicólogo tendrá que analizar en su situación particular.

Otro tema que no se ha abordado directamente es el fútbol femenino, el cual está creciendo, aunque actualmente representen el $4 \%$ del total de licencias en el fútbol español (MECD, 2017). A pesar de ello, el fútbol femenino es una oportunidad laboral para el psicólogo, el cual requiere una formación especializada, aspecto que podría ser extensible al resto de profesionales del área. Para un correcto desarroIlo de la actividad, se necesita adaptar el contenido de los entrenamientos y la competición al perfil físico, técnico, táctico y psicológico de la mujer (Martín, 2003). Como se puede observar en la presente revisión, el fútbol femenino requiere más investigación y experiencias profesionales.

El fútbol de aficionados es un nicho a atender por parte de los psicólogos/as, ya que representa un 13\% (124.000) de las licencias federativas (MECD, 2017), aunque debido a que no suelen tener un presupuesto amplio para este servicio, se podría presentar formatos que se ajusten a su situación deportiva, social y económica, como por ejemplo, a través de subvenciones, cuota mensual de las familias, pago privado por parte del cliente, etc.

Los árbitros de fútbol son una población que ha recibido una relativa atención por parte de los psicólogos/as (Alonso-Arbiol et al., 2008; Botella y Palacios, 2002; Brandão et al., 2011; Cantón, Gistain, Checa y León, 2012; De la Vega et al., 2017; Gómez y Botella, 2005; González, 2006; Marrero y Gutiérrez, 2002; Rodríguez et al., 2009). Principalmente, se ha estudiado, formado y entrenado variables psicológicas relacionadas con su desempeño arbitral (p. ej., concentración y comunicación). Con los avances tecnológicos y nuevas implantaciones en la Liga como es el uso del VAR (Capdevila, 2018), futuras investigaciones tendrán que ir encaminadas hacia su uso y múltiples situaciones asociadas al mismo (p. ej., cómo gestionar y comunicar los cambios arbitrales).

Y por último, la importancia de divulgar los avances cientiffcos-profesionales e investigar en Psicología del Deporte para el progreso y posicionamiento el área (Gómez-Espejo, Aroca et al., 2017; Martí, 2002). Por ejemplo, realización de ponencias y comunicaciones en cursos, seminarios y congresos, publicación de artículos, libros, informes, etc.

Para terminar, decir que es posible que el psicólogo del deporte no sea imprescindible en el fútbol, pero si cada vez más necesario para el logro de resultados, la mejora del rendimiento, la salud y educación de las personas implicadas en este deporte. A modo de comentarios finales relacionados con el ejercicio profesional del psicólogo en el fútbol y otros deportes, señalar que:

- La necesidad de una formación especializada del psicólogo en el área de la Psicología del Deporte y su continua actualización (reciclaje) para un mejor desempeño profesional (obtención de la acreditación de Experto en Psicología del Deporte del Consejo General de la Psicología de España). Además, posteriormente, sería positivo tener una sub-especialización en fútbol.

- Luchar contra el intrusismo y lograr que el psicólogo del deporte integre las profesiones del deporte desde un marco legal de referencia. El psicólogo del deporte debe de ser considerado un técnico del deporte, especialista en Psicología del Deporte.

- No auto-bloquear el acceso a posibles puestos de trabajo, como por ejemplo, que los clubes solo tengan psicólogos en prácticas (cursos, másteres, etc.) sin que haya algún psicólogo contratado, impidiendo en muchos casos que aumente la oferta laboral en esta especialidad. La responsabilidad de esta cuestión también es de las entidades formativas que lo puedan estar fomentando o reforzando.

- Realizar una Psicología del Deporte a partir de las necesidades reales de la propia actividad y población deportiva, la integración del conocimiento científico en la práctica profesional y definición del servicio ofertado e indicadores de eficacia.

- Mejorar el marketing del psicólogo del deporte, continuar naturalizando y promocionando la Psicología del Deporte y el rol del psicólogo en el fútbol, desarrollar la marca personal, el uso de redes sociales, medios de comunicación, etc. 
- El psicólogo del deporte es un bien necesario y rentable económicamente, en el que se necesita cuantifica esta cuestión (valor de mercado del jugador, número de jugadores que llegan al primer equipo, reducción del fracaso escolar, valoración social-deportiva....).

- El psicólogo del deporte debe de seguir trabajando como sabe, siendo sistemático, ordenado y, sobre todo, honrado con uno mismo y con los demás. Y desde esta perspectiva, exportar a los psicólogos/as del deporte de España a otros países dado el buen nivel que estos poseen.

\section{Referencias}

Andreu, M. D. (2003). Programa de entrenamiento psicológico y metodología de trabajo en un equipo de fútbol juvenil. Cuadernos de Psicología del Deporte, 3(2), 84-94.

Alonso, P. L. (02 de noviembre de 2017). El Málaga recurre a un psicólogo. Diario Sur. Recuperado de: http://malagacf.diariosur.es/malaga-20171102191327-nt.html

Alonso-Arbiol, I., Arratibel, N. y Gómez, E. (2008). La motivación del colectivo arbitral en fútbol: un estudio cualitativo. Revista de Psicología del Deporte, 17(2), 187-203.

Álvarez, M. S. y Chico, R. (2016). Experiencia del departamento de psicología en la escuela de fútbol base C.F. San José. Informació Psicológica, 112, 95-109.

Ardá, T., Maneiro, R., Rial, A., Losada López, J. L. y Casal, C. (2014). Análisis de la eficacia de los saques de esquina en la copa del mundo de fútbol 2010. Un intento de identificación de variables explicativas. Revista de Psicología del Deporte, 23(1), 165-172.

Barreira, D., Garganta, J., Castellano, J., Prudente, J. y Anguera, M. T. (2014). Evolución del ataque en el fútbol de élite entre 1982 y 2010: Aplicación del análisis secuencial de retardos. Revista de Psicología del Deporte, 23(1), 139-146.

Bohórquez, R. y Checa, I. (2017). Diseño y validez de contenido de una entrevista para la evaluación psicológica de porteros de fútbol. Revista de Psicología Aplicada al Deporte y al Ejercicio Físico, 1, E3. http://dx.doi.org/10.5093/rpadef2017a3

Botella, J. y Palacios, A. (2002). Limitaciones cognitivas en la apreciación del «fuera de juego» en fútbol. Revista de Psicología del Deporte, 17(2), 227-246.

Brandão, R., Serpa, S., Krebs, R., Araújo, D. y Machado, A. (2011). El significado de arbitrar: percepción de jueces de fútbol profesional. Revista de Psicología del Deporte, 20(2), 275-286.

Calahorro, F., Torres, G. y Lara, A. (2014). La percepción subjetiva de esfuerzo como herramienta válida para la monitorización de la intensidad del esfuerzo en competición de jóvenes futbolistas. Cuadernos de Psicología del Deporte, 14(1), 75-82. https://doi. org/10.4321/S1578-84232014000100009

Cantón, E. (2010). La Psicología del Deporte como profesión especializada. Papeles del Psicólogo, 37, 237-245.

Cantón, E. (2016). La especialidad profesional en Psicología del Deporte. Revista de Psicología aplicada al Deporte y al Ejercicio Físico, 1, E2. http://dx.doi.org/10.5093/rpadef2016a2
Cantón, E., Checa, I. y Budzynska, N. (2013). Estrategias de afrontamiento, optimismo y satisfacción con la vida en futbolistas españoles y polacos: un estudio preliminar. Revista de Psicología del Deporte, 22(2), 337-343.

Cantón, E., Gistain, C., Checa, I. y León, E. (2012). Programa de mediación psicológica con árbitros de fútbol. Revista Internacional de Ciencias Sociales y Humanidades SOCIOTAM, Vol. XXII, $n^{\circ} 2$ (Julio-Diciembre).

Capdevila, S. (04 de mayo de 2018).Todo lo que debes saber de la implantación del VAR en España: Preguntas con respuestas. Sport. Recuperado de: https://www.sport.es/es/noticias/ laliga/todo-que-debes-saber-implantacion-del-var-espana-preguntas-con-respuestas-6800893

Carmona, D., Guzmán, J. y Olmedilla, A. (2015). Efectos de un programa de formulación de objetivos y moldeamiento del pase en jóvenes jugadores de fútbol. Revista de Psicología del Deporte, 24(1), 81-88.

Carrascosa, J. (n.d.f). [Perfil Linkedin]. Recuperado de: https:// www.linkedin.com/in/jos\%C3\%A9-carrascosa-saber-competir-s-l-a4656863/

Casal, C., Losada, J. y Ardá, T. (2015). Análisis de los factores de rendimiento de las transiciones ofensivas en el fútbol de alto nivel. Revista de Psicología del Deporte, 24(1), 103-110.

Castellano, J. (2018). Relación entre indicadores de rendimiento y el éxito en el fútbol profesional. Revista Iberoamericana de Psicología del Ejercicio y el Deporte, 13(1), 41-49.

Castillo, I., Ramis, Y., Cruz, J. y Balaguer, I. (2015). Formación de entrenadores de fútbol base en el proyecto PAPA. Revista de Psicología del Deporte, 24(1), 131-138.

Castro, X. (29 de mayo de 2017). Unzué firma por dos años y vuelve al Celta con el cuerpo técnico de Lucho. La Voz de Galicia. Recuperado de: https://www.lavozdegalicia.es/noticia/gradario/2017/05/29/unzue-firma-dos-anos-vuelve-celta-cuerpo-tecnico-lucho/0003_201705G29D1991.htm

Centeno, A. (15 de febrero de 2018). Psicología para recuperar la fe. La Voz de Galicia. Recuperado de: https://www.lavozdegalicia.es/noticia/torremarathon/2018/02/10/psicologia-recuperar-fe/0003_201802G10P41992.htm

Chamorro, J., Torregrosa, M., Sánchez, D. y Amado, D. (2016). El fútbol dentro del campo y fuera de él: desafíos en la transición a la élite. Revista de Psicología del Deporte, 25(1), 81-89.

Chicau, C., Silva, C. y Palmi, J. (2012). Programa de Intervención psicológica para la optimización del concepto de equipo (team building) en jóvenes futbolistas. Revista de Psicología del Deporte, 27(1), 49-58.

Consejo General de la Psicología de España - CGPE (23 de junio de 2006). La Psicología marca gol. Infocop online. Recuperado de http://www. infocop.es/view_article.asp?id=904

Consejo General de la Psicología de España - CGPE (2018). Comisión Nacional de Acreditación Profesional. Recuperado de: http:// www.acreditaciones.cop.es/

Cruz, J., Boixadós, M., Torregrosa, M. y Mimbrero, J. (1996). ¿Existe un deporte educativo?: papel de las competiciones deportivas en el proceso de socialización del niño. Revista de Psicología del Deporte, 5(2), 111-134.

Cruz, J. y Cantón, E. (1992). Desarrollo histórico y perspectivas de la psicología del deporte en España. Revista de Psicología del Deporte, 7(1), 53-61. 
De la Vega, R. (2003). La importancia del entrenamiento de la concentración en el fútbol base: Una perspectiva aplicada. Cuadernos de Psicología del Deporte, 3(2), 67-82.

De la Vega, R., Carrasquilla, D., Ortiz, E., Ruiz-Barquín, R. y Armenteros, M. (2017). Influencia del tiempo de reacción y de movimiento en el rendimiento de árbitros de fútbol. Acción Psicológica, 14(1), 17-26. https://doi.org/10.5944/ap.14.1.19251

D’Amico, A., Cañizares, M. y Sánchez, B. P. (2017). La preparación psicológica de la selección nacional de fútbol de mayores de Venezuela (La Vinotinto): períodos 2001-2007 y 2008-2013. Cuadernos de Psicología del Deporte, 17(1), 59-72.

Duque-Ingunza, A. y Dosil, J. (2017). Variables que influyen en la transición deportiva: un estudio con futbolistas en activo y exfutbolistas de la primera división de la LFP. Cuadernos de Psicología del Deporte, 17(3), 13-24.

El Desmarque (30 de junio de 2016). El psicólogo del Depor no seguirá en el club. Recuperado de: http://coruna.eldesmarque.com/ deportivo/2335-el-psicologo-del-depor-no-seguira-en-el-club

El País (18 marzo de 2018). La Real Sociedad destituye a Eusebio y acepta la renuncia de Loren. Recuperado de: https://elpais.com/ deportes/2018/03/18/actualidad/1521408732_521872.html

Escolano-Pérez, E., Herrero-Nivela, M. L. y Echeverría-Expósito, R. (2014). La resolución de problemas como estrategia de enseñanza para el aprendizaje de la táctica ofensiva en la categoría pre-benjamín de fútbol 8. Revista de Psicología del Deporte, 23(1), 209-217.

Estrada, O, Silva, C., Pérez-Córdoba, E., Borrego, C. y Cantón, E. (2017). Intervención directa mediante biofeedback, para cambiar las conductas de desánimo de un portero de fútbol. Revista de Psicología del Deporte, 26(2), 131-136.

Galindo, A. y Arbinaga, F. (2017). Análisis bibliométrico de la Revista Iberoamericana de Psicología del Ejercicio y el Deporte (2006-2017). Revista Iberoamericana de Psicología del Ejercicio y el Deporte, 13(1), 33-40.

García, V. (14 de mayo de 2015). El fútbol español aporta 7.600 millones de euros y 140.000 puestos de trabajo. El Confidencial. Recuperado de: https://www.elconfidencial.com/deportes/futbol/liga/2015-05-14/el-futbol-espanol-genera-7-600-millonesde-euros-y-740-000-puestos-de-trabajo_794403/

García, J y Llames, R. (1992). Preparación psicológica de la selección olímpica de fútbol. Revista de Psicología del Deporte, 1(2), 63-67.

García-Naveira, A. (2010). El psicólogo del deporte en el alto rendimiento: aportaciones y retos futuros. Papeles del Psicólogo, 37(3), 259-268

García-Naveira, A. (2016). Área de psicología de la Real Federación española de atletismo: programa y desempeño profesional. Cuadernos de psicología del deporte, 16(1). 251-258.

García-Naveira, A. (2017). Entrenamiento psicológico para la mejora del autocontrol en un entrenador de fútbol. Acción Psicológica, 14(1), 27-42. https://doi.org/10.5944/ap. 14.7.19255

García-Naveira, A. y Jerez, P. (2012). Departamento de psicología del club Atlético de Madrid: filosofía, programación y desempeño profesional en el fútbol base. Cuadernos de Psicología del Deporte, 12(1), 111-120. https://doi.org/10.4321/S157884232012000100010

Getafe C. F. (2016). Escuela de padres. Recuperado de: http://www. getafecf.com/Noticia/tabid/137/Article/3723/Escuela-de-Padres.aspx
Gimeno, F., Sáenz, A. y Vicente, J. y Aznar, M. (2007). Deportividad y violencia en el fútbol base: un programa de evaluación y de prevención de partidos de riesgo. Revista de Psicología del Deporte, 16(1), 103-118.

Godoy-Izquierdo, D., Vélez, M. y Pradas, F. (2009). Nivel de dominio de las habilidades psicológicas en jóvenes jugadores de tenis de mesa, bádminton y fútbol. Revista de Psicología del Deporte, 18(1), 7-22.

Gómez, M. y Botella, J. (2005). El papel del feedback y la experiencia en la apreciación del 'fuera de juego' en fútbol. Revista de Psicología del Deporte, 14(1), 57-73.

Gómez-Espejo, V., Álvarez, I., Abenza, L. y Olmedilla, A. (2017). Análisis de la relación entre apoyo social y lesiones en futbolistas federados. Acción Psicológica, 14(1), 57-64. https://doi. org/70.5944/ap. 14.7.19261

Gómez-Espejo, V., Aroca, B., Robles-Palazón, F. y Olmedilla, A. (2017). Formación integral en la cantera del Real Murcia C.F.: tutorías psicológicas. Revista de Psicología Aplicada al Deporte $y$ al Ejercicio Físico, 2, E11. http://dx.doi.org/10.5093/rpadef2017a11

Gómez-Díaz, A., Pallarés, J., Díaz, A. y Bradley, P. (2013). Cuantificación de la carga física y psicológica en fútbol profesional: diferencias según el nivel competitivo y efectos sobre el resultado en competición oficial. Revista de Psicología del Deporte, 22(2), 463-469.

González, J. (2006). Aproximación a la formación del árbitro de fútbol: un ejemplo de modelo educativo. Cuadernos de Psicología del Deporte, 6(1), 37-51.

González, S., García, L. M., Pastor, J. C. y Contreras, O. (2011). Conocimiento táctico y toma de decisiones en jóvenes jugadores de fútbol (10 años). Revista de Psicología del Deporte, 20(1), 7997.

Hernández, D. (2016). La Psicología del Deporte en selecciones de fútbol de Costa Rica: experiencias y reflexiones. Revista de Psicología Aplicada al Deporte y al Ejercicio Físico, 1, E5. http:/l dx.doi.org/70.5093/rpadef2016a5

Interebook. (2017). Jose María Buceta. Recuperado de: http://www. interebook.com/autores/jose-maria-buceta.html

Lamoneda, J. y Huertas, F. (2017). Efectos de un programa de juego limpio aplicado a jóvenes futbolistas para la mejora de la deportividad. Revista Iberoamericana de Psicología del Ejercicio y el Deporte, 12(1), 107-119.

Levante (25 de enero de 2018). Muñiz recurre a un psicólogo deportivo. Recuperado de: http://www.levante-emv.com/ levante-ud/2018/01/16/muniz-recurre-psicologo-deportivo/7666591.html

Llames, R. (1999). Selección de jóvenes deportistas en fútbol. Revista de Psicología del Deporte, 8(2), 249-257.

Llopis, D. (n.d.e). [Perfil Linkedin]. Recuperado de: https://www. linkedin.com/in/david-llopis-goig-25667810/

Lorenzo, M y Bohórquez, R. (2013). Análisis de la percepción de los futbolistas acerca del proceso de retirada. Revista Iberoamericana de Psicología del Ejercicio y el Deporte, 8(1), 107-126.

Lorenzo, M. y Bueno, M. (2012). Recursos disponibles para la inserción sociolaboral tras la retirada deportiva de futbolistas. Revista de Psicología del Deporte, 21(1), 189-194.

Luengo, J. A. (n.d.d). [Perfil Linkedin]. Recuperado de: https://www. linkedin.com/in/jaluengolatorre/ 
Málaga C. F. (12 de febrero de 2018). Los padres vuelven a la escuela. Recuperado de: https://www. malagacf.com/noticia/lospadres-vuelven-a-la-escuela

Mancebo, A. (1992). Conversando con psicólogos del deporte profesional. Papeles del Psicólogo, 54. Recuperado de http://www. papelesdelpsicologo.es/resumen?pii=564

Marrero, G. y Gutiérrez, C. (2002). Las motivaciones de los árbitros de fútbol. Revista de Psicología del Deporte, 17(1), 69-82.

Martín, G. (2003). La mujer futbolista desde la perspectiva psicológica. Cuadernos de psicología del deporte, 3(2), 7-15.

Marín, K. (27 de noviembre de 2016). Eusebio, de ser despedido del Barça B a ambicionar "ganar la Liga" con la Real. El Confidencial. Recuperado de: https://www.elconfidencial.com/deportes/futbol/2016-11-27/eusebio-juan-carlos-alvarez-campi|lo-real-sociedad-barcelona-anoeta_1294215/

Martí, J. (2002). Fundamentos psicopedagógicos de aplicación en el Centro de Investigación y Desarrollo del Alto Rendimiento en Fútbol (CIDARF). Revista de Psicología del Deporte, 17(1), 125-135.

Martín, G. (2003). La mujer futbolista desde la perspectiva psicológica. Cuadernos de Psicología del Deporte, 3(2), 7-15.

Menchén, M. (02 de enero de 2017). Liga Santander: 2.900 millones en ingresos y menor brecha entre 'grandes y pequeños'. Palco 23. Recuperado de: https://www.palco23.com/clubes/ liga-santander-casi-3-000-millones-en-ingresos-y-recorte-a-lamitad-de-la-brecha-entre-grandes-y-pequenos.html

Miguelez, J. (26 de octubre de 1993). Gil contrata al psicólogo José Lorenzo González. El País. Recuperado de: https://elpais. com/diario/1993/10/26/deportes/751590002 850215.html

Ministerio de Educación, Ciencia y Deporte (MECD, 2017). Anuario de estadísticas deportivas 2017. Recuperado de: https://www. mecd.gob.es/servicios-al-ciudadano-mecd/dms/mecd/servicios-al-ciudadano-mecd/estadisticas/deporte/anuario-deporte/AED-2017/Anuario_de_Estadisticas_Deportivas_2017.pdf

Molleja, E. (n.d.b). [Perfil Linkedin]. Recuperado de: https://www. linkedin.com/in/eva-molleja-91662826/

Morelló, E. (n.d.c). [Perfil Linkedin]. Recuperado de: https:// www. linkedin.com/in/eduardo-morell\%c3\%B3-tom\%C3\%A7s-5616237b/

Moñino, L. (01 de septiembre de 2016). El método Lopetegui. El País. Recuperado de: https://elpais.com/deportes/2016/08/29/actualidad/1472499797_399264.html

Mora, J. (01 de agosto de 1993). Floro presenta al nuevo psicólogo del Real Madrid, Emilio Lamparero. El País. Recuperado de: https://elpais.com/diario/1993/08/01/deportes/744756008_850275.html

Mora, J. A., Zarco, J. A. y Blanca, M. (2001). Atención-concentración como entrenamiento para la mejora del rendimiento deportivo en jugadores profesionales de fútbol. Revista de Psicología del Deporte, 10(1), 49-65.

Morilla, M., Pérez-Córdoba, E., Gamito, J., Gómez, M., Sánchez, J. y Valiente, M. (2003). Planificación psicológica de la cantera del Sevilla F.C. S.A.D.: organización, funcionamiento y programa deportivo-formativo. Cuadernos de Psicología del Deporte, 3(2), 17-30.

Morilla, M., Utrilla, M., Antón, C. y Rivera, S. (2009). Proyecto de trabajo integrado deportivo-formativo con un equipo infantil del Sevilla fútbol club. En J. Dosil y A. Hernandez-Mendo (Coord.),
Psicología aplicada al futbol II, Actas del II Congreso se la Sociedad Iberoamericana de Psicología del Deporte (pp. 180-184). Torrelavega, España.

Navarrón, E., Godoy-Izquierdo, D., Vélez, M., Ramírez-Molina, M. y Jiménez-Torres, M. (2017). Implementación de una intervención psicológica en fútbol base, satisfacción subjetiva de los deportistas y experiencias de pasión, competencia percibida y compromiso deportivo en relación con la intención de práctica futura. Revista Iberoamericana de Psicología del Ejercicio y el Deporte, 12(1), 59-69.

Olmedilla, A. y Dominguez-Igual, J. (2016). Entrenamiento psicológico para la mejora de la atención y la autoconfianza en un futbolista. Revista de Psicología Aplicada al Deporte y al Ejercicio Físico, 1, E4. http://dx.doi.org/10.5093/rpadef2016a4

Olmedilla, A., García, C. y Garcés de los Fayos, E. (1998). Un análisis del papel profesional del psicólogo del deporte desde la percepción del entrenador de fútbol. Revista de Psicología del Deporte, 13, 95-111.

Olmedilla, A., García, C. y Martínez, F. (2006). Factores psicológicos y vulnerabilidad a las lesiones deportivas: un estudio en futbolistas. Revista de Psicología del Deporte, 15(1), 37-52.

Olmedilla, A., Ortega, E. y Abenza, L. (2007). Percepción de los futbolistas juveniles e influencia del trabajo psicológico en la relación entre variables psicológicas y lesiones. Cuadernos de Psicología del Deporte, 7(2), 75-87.

Olmedilla, A., Ortega, E., Andreu, M. y Ortín, F. (2010). Programa de intervención psicológica en futbolistas: evaluación de habilidades psicológicas mediante el CPRD. Revista de Psicología del Deporte, 19(2), 249-282.

Olmedilla, A., Ortín, F., Andréu, M. y Lozano, F. (2004). Formación en psicología para entrenadores de fútbol: una propuesta metodológica. Revista de Psicología del Deporte, 13(2), 247-262.

Olmedilla, A., Ortega, E., Ortín, F. y Andreu, M.D. (2008). Entrenamiento psicológico en fútbol base de élite: percepción de aplicabilidad e índices de satisfacción. Revista de Iberoamericana de Psicología del Ejercicio y el Deporte, 3(1), 31-46.

Ortiz, P. (2017). No todo es Balón. Implicando positivamente a los padres en la práctica del fútbol en etapa formativa. Revista de Psicología Aplicada al Deporte y al Ejercicio Físico, 2, E9. http:/l dx.doi.org/10.5093/rpadef2017a9

Pazo, C., Sáenz-López P. y Fradua, L. (2012). Influencia del contexto deportivo en la formación de los futbolistas de la selección española de fútbol. Revista de Psicología del Deporte, 21(2), 291299.

Prieto, J., Ortega, E., Garcés de los Fayos, E. y Olmedilla, A. (2014). Perfiles de personalidad relacionados con la vulnerabilidad del deportista a lesionarse. Revista de Psicología del Deporte, 23(2), 437-437.

Ramirez, P. y Rodriguez, Z. (2015). La intervención del psicólogo durante las concentraciones y competiciones. En A. García-Naveira y L. Locatelli (Eds.), Avances en Psicología del Deporte (pp. 293-312). Barcelona, España: Paidotribo.

Rivas, M. y Aragón, M. (2003). Intervención Psicológica en el fútbol base: un trabajo en el club América de México. Cuadernos de Psicología del Deporte, 3(2), 35-40.

Rodríguez, J. M. (2017). Personalidad, liderazgo y rendimiento en jugadores y entrenadores de fútbol y fútbol sala. Tesis doctoral. Universidad Autónoma de Madrid. 
Rodríguez, M., Salazar, W. y Arroyo, F. (2009). Juicio arbitral y desempeño cognoscitivo asociados con deshidratación en árbitros de fútbol. Revista de Iberoamericana de Psicología del Ejercicio y el Deporte, 4(1), 29-44.

Roffé, M. (2016). La preparación psicológica de la Selección Nacional Absoluta de Colombia para el Mundial de Fútbol Brasil 2014. Revista de Psicología Aplicada al Deporte y al Ejercicio Físico, 1, E3. http://dx.doi.org/10.5093/rpadef2017a2

Roffé, M. y Braco, M. (2003). Intervención Psicológica con la selección juvenil Argentina sub-16: Características "particulares" de los adolescentes inmersos en la singular dinámica del fútbol de élite. Cuadernos de Psicología del Deporte, 3(2), 41-50.

Roffé, R., De la Vega, R., Garcia-Mas, A. y Llinás, J. (2007). Las crisis durante el juego: el "gol psicológico" en el fútbol. Revista de Psicología del Deporte, 16(2), 227-240.

Ruiz-Barquín, R. (2015). Detección y selección de talentos deportivos desde una perspectiva psicológica. En A. García-Naveira y L. Locatelli (Eds.), Avances en Psicología del Deporte (pp. 245292). Barcelona, España: Paidotribo.

Sampedro, J. y Prieto, J. (2011). La territorialidad como factor asociado a la ventaja de jugar en casa. Un estudio comparativo por regiones en la liga de fútbol y en la liga de fútbol sala de España. Motricidad. European Journal of Human Movement, 26, 93-104.

Sampedro, J. y Prieto, J. (2012). El efecto de marcar primero y la ventaja de jugar en casa en la liga de fútbol y en la liga de fútbol sala de España. Revista de Psicología del Deporte, 21(2), 301-308
Sánchez-Gombau, M. C. y Guillén, F. (2015). Selección y scouting de deportistas. En A. García-Naveira y L. Locatelli (Eds.), Avances en Psicología del Deporte (pp. 229-244). Barcelona, España: Paidotribo

Sans, G. (30 de julio de 2014). Joaquín Valdés: Psicólogo hasta en las ruedas de prensa. Mundo Deportivo. Recuperado de: http://www. mundodeportivo.com/20140730/fc-barcelona/joaquin-valdes-psicologo-hasta-en-las-ruedas-de-prensa_54412604084.htm

Soriano Recio, J. (n.d.a). [Perfil Linkedin]. Recuperado de: https:// www.linkedin.com/in/javier-soriano-recio-2b380134/

Suso, R. (12 de julio de 2017). El Athletic prescinde de su psicóloga después de 21 años. Mundo Deportivo. Recuperado de: http://www.mundodeportivo.com/futbol/ athletic-bilbao/20170712/424079839868/athletic-psicologa-ruiz-de-ona-despedida-21-anos.html

Vales, A., Blanco, H., Areces, A. y Arce, C. (2015). Perfiles de rendimiento de selecciones ganadoras y perdedoras en el Mundial de fútbol Sudáfrica 2010. Revista de Psicología del Deporte, 24(1), 111-118.

Villalonga, T., Garcia-Mas, A., De las Heras, R., Buceta, C. y Smith, R. (2015). Instauración y tareas de un servicio de psicología del deporte en un club de fútbol profesional. Revista de Psicología del Deporte, 24(2), 319-326.

Vives, L. y Garces de los Fayos, E. (2003). Intervención psicológica en un club de fútbol base: propuesta de un sistema de actuación psicológica desde sus diversas áreas. Cuadernos de Psicología del Deporte, 3(2), 51-66. 